\title{
Análisis de indicadores de innovación para una muestra de empresas manufactureras de Montería (Colombia)*
}

\author{
Recibido: 14 de marzo de 2019 • Aprobado: 23 de mayo de 2019 \\ https://doi.org/10.22395/seec.v22n52a3 \\ Orlando Mauricio Lorduy Herrera**
Jaime Eliécer Rangel Bolaños
}

RESUMEN

Este trabajo busca caracterizar los procesos de innovación generados por las empresas manufactureras de la ciudad de Montería en el departamento de Córdoba, Colombia, tomando como referente la teoría neoevolucionista, la cual entiende la motivación para innovar como un proceso schumpeteriano de destrucción creativa. Para alcanzar este objetivo se recurre a un instrumento tipo encuesta que sigue los lineamientos establecidos por los manuales de Oslo, de Bogotá, y las encuestas de innovación (EDIT) implementadas por el DANE en Colombia. Se encuentra que las empresas manufactureras de la ciudad son innovadoras en sentido amplio, esgrimen estrategias de carácter defensivo y realizan actividades tecnológicas que representan innovaciones, más para la empresa o para el mercado local.

\section{PALABRAS CLAVE}

Innovación; cambio técnico; destrucción creativa; evolucionismo; empresas manufactureras.

\section{CLASIFICACIÓN JEL}

O31, O32; O33; O14; L21

\section{CONTENIDO}

Introducción; 1. La innovación y el crecimiento; 2. La innovación desde lo local; 3. La evidencia; 4. Conclusiones; Bibliografía.

* Este artículo es resultado de la investigación realizada para la Universidad Pontificia Bolivariana en su convocatoria para profesores investigadores internos, CIDI 2016, grupo Esder de la Escuela de Economía, Administración y Negocios, clasificado en categoría C por Colciencias.

* Economista, Universidad Externado de Colombia, Bogotá, Colombia. Magíster en Ciencias Económicas, Universidad Santo Tomás, Bogotá, Colombia. Profesor, consultor e investigador, Universidad Pontificia Bolivariana, Montería, Colombia. Correo electrónico: olorduy@hotmail.com

** Economista, Universidad Pontificia Bolivariana, Montería, Colombia. Estudiante de Maestría en Economía, Universidad del Norte, Barranquilla, Colombia. Profesor asistente Facultad de Economía, Universidad Pontificia Bolivariana, Montería, Colombia. Correo electrónico: jaime.rangelb@upb.edu.co 


\title{
ANALYSIS OF THE INNOVATION INDICATORS OF A SAMPLE OF MANUFACTURER COMPANIES OF MONTERÍA (COLOMBIA)
}

\begin{abstract}
This work pursuit a characterization of the innovative processes generated by the manufacturing companies of the city of Montería in the department of Córdoba, Colombia, taking as theoretical reference the neo-evolutionary theory, which understands the motivation for innovation as a Schumpeterian process of creative destruction. For reaching this goal, a poll-type tool is used following the guidelines established by the Oslo and Bogotá manuals, as well as the innovation polls (EDIT) implemented by the Dane in Colombia. We found that manufacturing companies in this city are innovative in a wide way, they wield defensive strategies and undergo technological activities that result in innovation to the company as to the local market.
\end{abstract}

\section{KEYWORDS}

Innovation; technical change, creative destruction, evolutionism; handcrafting companies.

JEL CODE

O31, O32; O33; O14; L21

\section{CONTENTS}

Introduction; 1. Innovation and growth; 2. Innovation from the local; 3. Evidence; 4. Conclusions; Bibliography.

\section{ANÁLISE DE INDICADORES DE INOVAÇÃO PARA UMA AMOSTRA DE EMPRESAS MANUFATUREIRAS DE MONTERÍA (COLÔMBIA)}

\section{RESUMO}

Este trabalho caracteriza os processos de inovação gerados pelas empresas manufatureiras da cidade de Montería, no estado de Córdoba, Colômbia, tendo como referencial teórico a teoria neoevolucionista, a qual entende a motivação para inovar como um processo "schumpeteriano" de destruição criativa. Para atingir esse objetivo, recorre-se a um questionário que segue os lineamentos estabelecidos pelos manuais de Oslo, de Bogotá, e as pesquisas de inovação (EDIT) implantadas pelo Departamento Administrativo Nacional de Estatística da Colômbia. Verifica-se que as empresas manufatureiras dessa cidade são inovadoras em sentido amplo, desenvolvem estratégias de caráter defensivo e realizam atividades tecnológicas que representam inovações, mais para a empresa ou para o mercado local.

\section{PALAVRAS-CHAVE}

Inovação; mudança técnica; destruição criativa; evolucionismo; empresas manufatureiras.

\section{CLASSIFICAÇÃO JEL}

O31, O32; O33; O14; L21

\section{CONTEÚDO}

Introdução; 1. Inovação e crescimento; 2. A inovação a partir do local; 3. A evidência; 4. Conclusões; Bibliografia. 


\section{INTRODUCCIÓN}

La innovación se ha constituido en el caballo de batalla para las administraciones nacionales y regionales, sobre todo cuando se quieren justificar las bondades que tienen las estrategias de crecimiento adoptadas en los planes de desarrollo (Godín citado por Albornoz, 2009, p.10). Sin embargo, en Colombia, las políticas relacionadas con la ciencia, la tecnología y la innovación (CTi), ya bien entrado el siglo XXI, no parecen haber dado los resultados esperados en materia de integración sistemática de los actores a lo que, actualmente, se conoce como el Sistema Nacional de Ciencia, Tecnología e Innovación (SNCTi) ${ }^{1}$.

En efecto, a pesar de que los referentes teóricos y empíricos, desde la vertiente del crecimiento endógeno, hasta las teorías heterodoxas basadas en la destrucción creativa de Schumpeter y el evolucionismo de Nelson y Winter, reconocen la importancia de la innovación y de sus actividades conexas en la generación de las sinergias necesarias para mejorar la productividad, la competitividad, el ingreso per cápita y el bienestar, el país muestra un atraso inquietante frente a algunos de sus pares de similar grado de desarrollo y los esfuerzos realizados hasta ahora se muestran insuficientes. Peor aún, tales esfuerzos parecen diluirse en pequeños logros que no trascienden a nivel nacional, ni a nivel regional².

En Colombia, la recopilación de las estadísticas asociadas con las actividades generales relacionadas con la CTi, ha correspondido principalmente al Observatorio Colombiano de Ciencia y Tecnología (OCyT), mientras que la medición cuantitativa y la descripción cualitativa de las actividades empresariales asociadas con el esfuerzo innovador le conciernen al Departamento Administrativo Nacional de Estadística (DANE). La información que proporciona la Encuesta de desarrollo e innovación tecnológica (EDIT) que realiza este último, es de utilidad limitada si lo que se quiere es caracterizar la dinámica del cambio técnico y la innovación del sector manufacturero en los departamentos más rezagados y en las ciudades intermedias del país.

1 Ciertamente, el plan de desarrollo del primer gobierno del presidente Santos (2010-2014), llamó a este componente prioritario la "locomotora de la innovación", sin embargo, los análisis de los medios económicos especializados sugieren que, a 2018, sus objetivos se cumplieron en baja proporción (Consejo Privado de Competitividad, 2018; Gómez y Mitchell, 2014). Así mismo, el Departamento Nacional de Planeación (DNP, 2015b) diagnosticaba con respecto al SNCTi que "el principal problema (...) es que el país y sus regiones no han logrado superar los retos que persisten en términos de capacidades de los actores, y de falencias en los resultados y eficiencia de los recursos invertidos".

2 Quizás, las dos excepciones las constituyan Bogotá y Medellín: la primera, con todas las posibilidades que genera la concentración de actividades en su área de influencia (capital humano, empresas, gremios, universidades, centros de investigación y posibilidades financieras); la segunda, impulsada por el buen manejo de los recursos, el talante emprendedor, la confianza entre los actores del sistema y la buena gestión pública (Gómez y Mitchell, 2014, p. 80). 
Para el departamento de Córdoba y su capital Montería, no se cuenta con información específica acerca del comportamiento del empresariado frente al reto de la innovación desde el punto de vista de su dinámica interna y externa. También es cierto que existen ciertas restricciones al acceso de la información departamental y municipal de las encuestas EDIT (microdato), como consecuencia del escaso tejido industrial existente en muchos de estos territorios.

Este trabajo intenta suplir la carencia de información referente a la caracterización de la dinámica innovadora de las empresas manufactureras de tamaño pequeño y mediano, con domicilio principal en la ciudad de Montería, mediante la aplicación de una encuesta piloto de carácter limitado. Este esfuerzo pionero es de gran importancia para contribuir a la comprensión de las idiosincrasias empresariales locales y de las dinámicas de participación, integración y colaboración. Con ello, se les da a los hacedores de políticas los insumos necesarios para una mejor estructuración de lo que, a futuro, debería de constituirse en un ecosistema regional de CTi, con la participación activa de todos los actores de las cadenas productivas, del sistema financiero, del sector público, de las universidades, de los centros de pensamiento y de desarrollo tecnológico.

El documento consta de cuatro secciones: en la primera se presentan los referentes teóricos conceptuales más relevantes; en la sección dos se recoge la evidencia para Colombia; en la tercera se especifica la metodología general del ejercicio junto con los resultados; finalmente, en la sección cuatro se sintetizan las conclusiones y recomendaciones.

\section{LA INNOVACIÓN Y EL CRECIMIENTO}

La literatura que relaciona el crecimiento económico con el cambio técnico, el desarrollo de la innovación y sus interrelaciones es amplia, y los resultados positivos en aquellas sociedades en las que se ha insertado exitosamente, están por fuera de toda consideración ${ }^{3}$.

Desde la óptica de la escuela neoclásica moderna, los modelos de crecimiento endógeno incorporan el cambio técnico como parte de la función de producción de diversas maneras, utilizando supuestos simplificadores que facilitan la interpretación matemática pero los alejan de la realidad, del entendimiento de los procesos de cambio técnico desde adentro, es decir, desde el mismo momento en que se toman

3 Algunas de las principales modelaciones del crecimiento con cambio técnico endógeno (incorporado en la función de producción), se recogen en Aghion y Howitt (1999) y en Barro y Sala-i-Martin (2004). Romer (1994) realiza un recuento completo de los modelos pioneros del crecimiento endógeno. Otros trabajos relevantes incluyen a Romer (1990), Barro (1991) y más recientemente Jones y Romer (2009). 
las decisiones empresariales (Nelson y Winter, 1982; Nelson 1995, p. 68; Fagerberg, 2005, p. 3). En este sentido, los complejos desarrollos de optimización dinámica suponen la existencia de mercados competitivos a pesar de que gran parte de estos están cada vez más alejados del ideal de la perfecta información y de la toma de precios por parte de los oferentes y demandantes. Es así como la disposición y la disponibilidad innovadoras no se tienen en cuenta para la selección de la técnica de producción, para la transformación productiva o para las actividades conexas, y generalmente se considera que los efectos spillovers en los mercados son suficientes para igualar la competencia en el largo plazo (Aghion y Howitt, 1999, p. 37).

Aunque el paradigma neoclásico sigue siendo el favorito para relacionar el crecimiento económico con diferentes formas de innovación tecnológica, los desarrollos desde el punto de vista heterodoxo han sido copiosos, especialmente los que se derivan del proceso de cambio técnico desde la visual schumpeteriana, según la cual este está justificado porque permite generar rentas de monopolio transitorias a aquellas empresas que lo realizan. En consecuencia, los empresarios que sobreviven en los mercados son los que innovan permanentemente, en un proceso conocido como de destrucción creativa (Schumpeter, 1946). En ese orden de ideas, hay empresarios que innovan radicalmente y empresarios que innovan incrementalmente, aquellos que no lo hacen tienden a desaparecer (podemos llamar a los primeros innovadores en sentido estricto, y al resto seguidores). La innovación se constituye así, en una actividad esencial para entender las estructuras de los mercados modernos y sus interacciones en un marco de incertidumbre. En palabras de Katz (1990, p. 59), el enfoque de Schumpeter contribuye a entender el cambio técnico como un resultado complejo de interacciones dentro del sistema económico.

Se entiende entonces que la innovación, como proceso colectivo, también depende de lo que suceda a nivel macroeconómico y mesoeconómico, del marco institucional, del entorno social y religioso, del sistema educativo, etc. (Katz, 2000). Es decir, de un conjunto de variables que suelen ser exógenas al proceso de toma de decisiones por parte del empresario. De esta manera, es posible identificar sociedades que son más proclives al desarrollo de actividades de CTi que otras. Lo anterior conlleva a tratar el proceso de innovación como un sistema de interrelaciones complejo que se traduce en sistemas nacionales y regionales dinámicos, sociales, autosostenibles y reproducibles (Lundvall, 2016).

La visión evolucionista del cambio técnico se constituye en un avance frente a las ideas schumpeterianas originales. Como su nombre lo indica, esta recoge elementos de la teoría de la selección natural de Darwin, entendiendo a la innovación como un proceso de aprendizaje y asimilación complejo y dinámico que debe ser 
analizado y comprendido desde la interdisciplinariedad (Fagerberg, 2005, p. 3). Así, la supervivencia empresarial implica que el cambio y el desequilibrio constituyen la regla y no la excepción. Es un proceso prolongado y riesgoso que se caracteriza por permanentes búsquedas y arreglos sobre un proceso de prueba y error que no todo empresario está dispuesto a asumir. Bajo esta óptica, los agentes maximizan en un entorno de incertidumbre e información incompleta y actúan con racionalidad acotada. "Como quiera, las firmas son vistas como poseedoras de diferentes habilidades, procedimientos, y reglas de decisión que determinan su quehacer dadas ciertas condiciones externas. También emplean diferentes procedimientos de exploración para descubrir, considerar y evaluar posibles cambios en su forma de hacer las cosas" (Nelson y Winter, 1982, p. 206).

Los últimos desarrollos de la teoría evolucionista se enfocan más en las interrelaciones complejas que caracterizan los mercados modernos, y en las estructuras sociales e institucionales que sostienen (o impiden) el crecimiento innovador. Este neoevolucionismo concibe la innovación como un proceso sistémico interdependiente en el que interactúan las empresas (como parte de una industria), y las instituciones políticas, económicas y sociales que la soportan y le dan vida como sistema nacional, primero, y como sistemas regionales, después (Nelson, 2008). La innovación se constituye en la base del entendimiento de las dinámicas de innovación diferenciadas, no solamente a nivel de país, sino también a nivel de regiones dentro de los países. Más importante aún, dichas dinámicas son determinantes para entender los diferenciales en el crecimiento económico que tanto preocupan a los hacedores de políticas en particular, y a la sociedad en general.

\section{LA INNOVACIÓN DESDE LO LOCAL}

De acuerdo con los planteamientos teóricos expuestos en la sección anterior, la innovación, entendida como un proceso social, debe ser estudiada desde adentro (empresa), sin perder de vista las particularidades idiosincrásicas del entorno en donde la desarrolla (Aghion y Howitt, 1999, p. 1). La innovación se concibe, entonces, desde las decisiones del empresario tomando en cuenta todas las restricciones existentes y el grado de desarrollo institucional. Esto es especialmente importante para un país en desarrollo como el nuestro y para sus regiones, dado que las actividades de Investigación y Desarrollo (I+D) y de CTi son limitadas y segmentadas (Esguerra, Castro y González, s. f.; González y Ramírez, s. f.; Albornoz, 2009, p. 19).

Para Colombia, se han elaborado varios estudios en la búsqueda de la caracterización de los diversos aspectos involucrados en el sistema de CTi, sus actores y actividades conexas. Esto se ha hecho con base en lo establecido en el Manual de Bogotá (2001), que a su vez es una adaptación para Latinoamérica y el Caribe, de los manuales Frascati (quinta edición) y de Oslo (primera edición), de la OCDE (Organización 
para la Cooperación y el Desarrollo Económicos). Es así como la primera encuesta tecnológica se realizó en 1996 (Encuesta de Desarrollo Tecnológico -EDT-, Departamento Nacional de Planeación y Colciencias), pero solamente tuvo continuidad desde 2006 (EDIT, cuya última versión es la EDIT VIII, 2015-2016).

Entre los trabajos que han utilizado la EDT y EDIT para el análisis de la innovación y sus interrelaciones en un sentido amplio se encuentran: Durán et al. (1998, 2000), Bonilla (2000), Vargas y Malaver (2004), Langebaek y Vásquez (2007), Anlló y Suárez (2008), Anlló, Suárez y De Angelis (2009), Gómez (2009), Arbeláez y Parra (2011), Bitrán, Benavente y Maggi (2011), Gómez (2011), Marins, Anlló y Schaaper (2013), Torres, Castaño y Arias (2015). Estos estudios muestran la realidad de la innovación, principalmente desde un punto de vista agregado. En Durán et al. (1998), se presentan algunas comparaciones departamentales, tomando como referentes a Bogotá y a los principales departamentos del país, como son Antioquia, Valle, Cundinamarca y Atlántico. El trabajo de Durán et al. (2000) se enfoca en el análisis regional de los resultados de la EDT I, y se constituye en el primer referente para diferenciar las capacidades innovadoras de las regiones.

En general, las conclusiones expresadas en estos estudios coinciden en indicar que la innovación ha sido marginal en el comportamiento empresarial típico en Colombia y en sus departamentos, por lo que las empresas innovadoras en sentido estricto se constituyen en una minoría, mientras que la innovación en un sentido amplio es más común, especialmente aquella referente a la organización y a la comercialización (tecnologías blandas). Según Bitrán, Benavente y Maggi (2011, p. 25), $11,8 \%$ de las empresas colombianas eran innovadoras en sentido estricto (EDIT IV); asimismo, el 73,6 \% de las empresas manufactureras pertenecían a la categoría de no innovadoras (EDIT VI). Este hallazgo se puede asociar con las estrategias empresariales de carácter defensivo que no favorecen el desarrollo de la innovación (Jaramillo, Lugones y Salazar, 2001; Vesga, 2008, p. 9). Lo mismo puede afirmarse de las pymes, para las cuales, Torres, Castaño y Arias (2015) encontraron que solo el 33,25\% pertenecían al grupo innovador propiamente dicho, mientras que el 52,99\% hacía parte del grupo de empresas rezagadas (EDIT IV).

Si bien las EDIT son un excelente instrumento para caracterizar la innovación a nivel agregado, tienen limitaciones funcionales cuando se quiere estudiar el microdato a nivel departamental, especialmente para aquellos departamentos que no tienen tradición manufacturera, como es el caso del departamento de Córdoba. Esto se debe a que es fácil identificar a las empresas encuestadas por actividad económica, con lo cual el secreto estadístico puede quedar en entredicho por la facilidad de asociación, tal como se explica en la sección 3. 
Para conocer más a fondo las problemáticas locales y regionales, la Cámara de Comercio de Bogotá, en asocio con la Universidad Javeriana, ha realizado dos ejercicios denominados Encuesta de innovación Bogotá y Cundinamarca (2005 y 2010), con una base conceptual similar a la de las EDIT. El análisis de los resultados más reciente fue preparado por Vargas y Malaver (2013). Los autores señalan que la mayoría de empresas consultadas pertenecen a las categorías de potencialmente innovadoras ( $31,1 \%)$ o no innovadoras ( $28,7 \%)$, y unas pocas son innovadoras en sentido estricto (9,2\%). En el global, el porcentaje de empresas que hacen innovaciones y realizan actividades de I+D conducentes a obtener innovaciones de alcance nacional disminuyó de $49 \%$ a $40 \% 4$.

Otros trabajos relevantes que no toman como base estadística e informativa las encuestas anteriormente mencionadas, incluyen a González y Ramírez (s. f.) y Esguerra, Castro y González (s. f.), que analizaron la competitividad en 24 departamentos de Colombia, teniendo en cuenta los componentes tecnología, innovación y capital humano, entre otros. Estos autores, utilizaron la clasificación propuesta por Gera y Masse (1996) para determinar la intensidad de uso del conocimiento por grupos de industrias, encontrando que, entre 1981 y 2002, 17 departamentos perdieron participación manufacturera en su producto interno, incluyendo 12 en los cuales la participación de industrias medias de conocimiento ha caído significativamente (Córdoba, Antioquia, Magdalena, Valle y Tolima, entre otros). Este resultado se puede considerar como un indicio de desindustrialización y de pérdida de la dinámica innovadora para la mayoría de los departamentos de Colombia, lo cual puede estar en contraposición con la búsqueda de sistemas regionales de innovación generadores de valor agregado, fuertes y dinámicos.

En resumen, la actividad investigativa en lo referente al estudio de la innovación se ha incrementado a medida que aumenta la disponibilidad de información transversal y longitudinal, principalmente a nivel de sistema nacional. En términos de caracterización y comparación regional todavía no hay muchos estudios, por lo que este ejercicio representa una buena contribución al entendimiento de la realidad regional de nuestro país.

Finalmente, no está de más advertir que el grado de especificidad que se requiere actualmente para comprender el problema interdisciplinario del cambio técnico y organizacional, ha conducido al surgimiento de nuevos referentes que

Esto es, no solo preocupante, sino también, probablemente indicativo de lo que ocurre en el resto de departamentos (con importantes diferencias intrarregionales), más teniendo presente que la participación de Bogotá y Cundinamarca en el producto manufacturero nacional fue del 31, 07 \% en el 2017 y fue muy similar para los años de análisis (DANE). 
permiten la construcción y aplicación de instrumentos concretos que contribuyen al entendimiento, cada vez más exacto, de la innovación y sus actividades conexas (por ejemplo, los manuales de Santiago (2007), Lisboa (2009) Antigua (2015), Valencia (2017), entre otros).

\section{LA EVIDENCIA}

Este trabajo fue desarrollado utilizando un instrumento de recolección de datos tipo encuesta, con énfasis cualitativo, dirigido a la totalidad de las empresas manufactureras con domicilio en la ciudad de Montería, matriculadas en la Cámara de Comercio. Todas ellas pertenecientes a la categoría de pequeña y mediana empresa5. La información se recolectó durante el año 2017 y el método de recolección fue el de la entrevista directa con el propietario o administrador del establecimiento. El cuestionario sigue los lineamientos metodológicos establecidos por el DANE y considera al empresario como el principal impulsor de las actividades relacionadas con la tecnología y la innovación en un sentido amplio (estrategias, incentivos, barreras) ${ }^{6}$.

La implementación del instrumento encontró un obstáculo insalvable en la baja tasa de participación de los empresarios, de hecho, de 38 empresas manufactureras registradas en la Cámara de Comercio de Montería, solamente 10 contestaron la encuesta, lo que da una tasa de participación voluntaria del 26,3\%. Al respecto, Albornoz (2009, p. 17) indica que la tasa de contestación voluntaria a nivel internacional para este tipo de encuestas es del $50 \%$ aproximadamente. Para Colombia la tasa de respuesta para las encuestas EDIT 2005 y 2007 fue superior al 50 \% (Marins, Anlló y Schaaper, 2012). En este caso, se puede generar una comprensible incertidumbre frente a la generalización de los resultados. Sin embargo, el conocimiento del medio permite hacer acto de fe en que la muestra es representativa de lo que ocurre a nivel local e incluso regional, así no lo sea en un sentido estadístico más estricto.

No obstante, lo precedente tuvo dos consecuencias indeseadas pero inevitables para esta investigación: la primera es que se omitió la información sobre la actividad económica que realiza la empresa (clasificación CIIU rev. 3 o 4) en aras de la preservación del secreto estadístico; la segunda, es que se imposibilitó la realización de cualquier modelación econométrica de corte transversal, para evitar

5 La Ley 590 de 2000 estipula que las empresas pequeñas son las que tienen activos entre 501 SML y 5.000 SML o tienen entre 11 y 49 trabajadores, las empresas medianas son aquellas que tienen activos entre 5.001 SML y 15.000 SML o tienen entre 50 y 200 trabajadores, mientras que las empresas grandes son aquellas que cuentan con activos superiores a 15.001 SML o tienen más de 200 trabajadores.

6 El cuestionario no se incluye por cuestiones de espacio, se puede solicitar a los correos de los autores. 
la presencia de sesgos estadísticos que conlleven resultados espurios. El único dato significativo que se puede aportar es que todas las empresas pertenecen a las categorías de industrias bajas y medias en intensidad de uso del conocimiento según la clasificación ISIC (International Standard Industrial Classification).

Sin perder de vista lo anterior, se procedió al análisis de resultados. En la tabla 1 se presenta la información estadística general para las empresas participantes en esta investigación preliminar (transformada en una prueba piloto). Se observa que la diferencia entre las empresas participantes es marcada en todas las variables, desde el número de empleados, pasando por el tiempo de permanencia en el mercado, el número de competidores identificados, hasta las variables monetarias sobre ingresos por ventas y patrimonio registrado en libros.

Tabla 1. Información general consolidada sobre las empresas participantes en la prueba

\begin{tabular}{lccccc}
\hline & $\begin{array}{c}\text { Número de } \\
\text { empleados }\end{array}$ & $\begin{array}{c}\text { Número de años } \\
\text { en ercado }\end{array}$ & $\begin{array}{c}\text { Competidores en el } \\
\text { mercado }\end{array}$ & $\begin{array}{c}\text { Ingresos aproximados } \\
\text { porventas (2016) }\end{array}$ & $\begin{array}{c}\text { Patrimonio registrado } \\
\text { (2016) }\end{array}$ \\
\hline Máximo & 100,00 & 57,00 & 10,00 & $\$ 24.000 .000 .000$ & $\$ 7.673 .056 .000$ \\
\hline Mínimo & 12,00 & 3,00 & 2,00 & $\$ 656.000 .000$ & $\$ 365.682 .755$ \\
\hline Promedio & 43,33 & 22,55 & 4,87 & $\$ 7.648 .000 .000$ & $\$ 3.152 .898 .338$ \\
\hline Desviación & 28,37 & 18,80 & 2,47 & $\$ 8.974 .903 .973$ & $\$ 2.982 .706 .156$ \\
\hline Mediana & 35,00 & 16,00 & 5,00 & $\$ 2.700 .000 .000$ & $\$ 1.208 .138 .750$ \\
\hline
\end{tabular}

Fuente: elaboración propia según datos suministrados por la Cámara de Comercio de Montería (2016).

El análisis gráfico permitió realizar una caracterización general con algunos elementos concordantes con los resultados alcanzados anteriormente (ver sección 2). En primera instancia, se encontró que el 78 \% de las empresas manufactureras que participaron en esta prueba venden sus productos principalmente a nivel local y regional (entendiendo regional al área que incluye a los departamentos de Córdoba y Sucre, el Urabá y el Bajo Cauca antioqueños), y solamente el 22 \% tiene participación en el mercado nacional, este resultado es compatible con la carencia de iniciativa para la ampliación de mercados con la que usualmente se identifica a los empresarios de la región, empresarios tradicionales, conformes con la situación de sus empresas en el mercado y reticentes al riesgo?

\footnotetext{
7 Según la EDIT VIII, en Colombia solamente el 11,9\% de las empresas manufactureras se puede considerar innovadora de producto para el mercado nacional y un 2,2 \% para el mercado internacional.
} 
De otro lado, ante la indagación de cuáles actividades de innovación (ACTI) ha desarrollado la empresa en los últimos cinco años, el 80 \% estableció que la adquisición de tecnología incorporada al capital (ATIK) era su actividad principal, seguida de capacitaciones y diseño, ambas con el $70 \%$, cambios organizacionales (50\%), adquisición de tecnología no incorporada al capital (Atnik) y comercialización (40 \% para ambas opciones). Solamente el $20 \%$ de las empresas afirmó realizar actividades específicas de I+D. Se puede decir entonces que todas las empresas desarrollan algún tipo de innovación en sentido amplio, pero la innovación en sentido estricto es una de las tareas pendientes para los empresarios de la ciudad. La tabla 2 muestra la distribución porcentual por actividad innovadora. Es de destacar que el promedio para I+D es de solamente 5,56\%, resultado acorde con los resultados alcanzados por Arbeláez y Parra (2011).

Tabla 2. Distribución de recursos por ACTI (\%)

\begin{tabular}{cccccccc}
\hline & I+D & ATIK & Atnik & Capacitación & $\begin{array}{c}\text { Cambios } \\
\text { organizacionales }\end{array}$ & Diseño & Comercialización \\
\hline Máximo & 30,00 & 90,00 & 100,00 & 30,00 & 30,00 & 70,00 & 40,00 \\
\hline Mínimo & 0,00 & 0,00 & 0,00 & 0,00 & 0,00 & 0,00 & 0,00 \\
\hline Promedio & 5,56 & 38,56 & 12,33 & 9,89 & 7,78 & 14,22 & 11,67 \\
\hline Desviación & 11,30 & 36,52 & 32,93 & 10,37 & 12,86 & 24,57 & 15,41 \\
\hline
\end{tabular}

Fuente: elaboración propia.

El gráfico 1 parece confirmar la apreciación anterior. En él se muestra la información consolidada para las ACTI, se observa que la tasa de respuesta para la actividad de I+D sobre el total fue solo del $8 \%$.

Cuando se les solicitó a los empresarios que distribuyeran porcentualmente los recursos asignados a cada actividad, se encontró que el 40 \% destinó 50 \% o más a ATIK, y solamente un 10 \% destinó recursos iguales o mayores al 50 \% a Atnik y a diseño. Otra lectura de los datos indica que el $70 \%$ de las empresas realiza actividades ATIK, el 60 \% realiza actividades de capacitación, el 50 \% realiza actividades de diseño, el $40 \%$ dedica recursos a actividades Atnik, organizacionales y de comercialización. Solamente el 20 \% dice realizar actividades I+D. Al respecto, Esguerra, Castro y González (s.f., p. 39) señalan que para Colombia "Los niveles de innovación tecnológica y de intensidad en la utilización del conocimiento, en la industria, son bajos; concentrados en su mayoría en las industrias medias". A manera de referencia, en la EDIT VIII se identifica que la adquisición de maquinaria y equipo es la ACTI con mayor monto de inversión asignado, conforme con lo puntualizado para Montería. 
Gráfico 1. ACTI que ha desarrollado la empresa en los últimos cinco años (participación porcentual en el total de actividades)

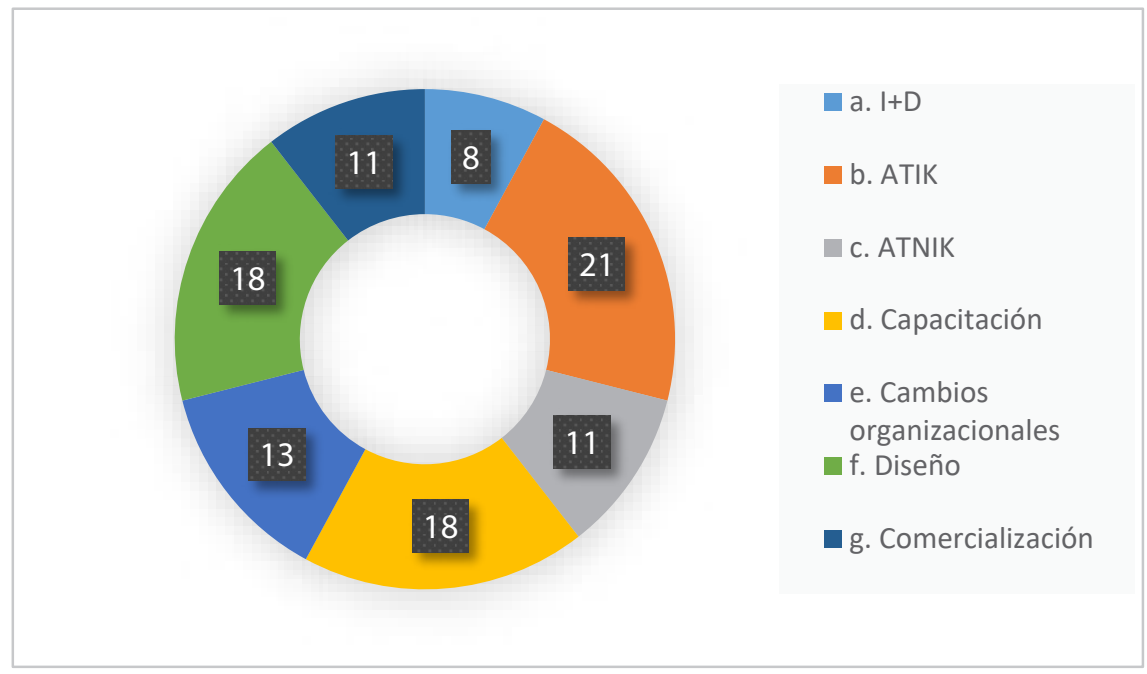

Fuente: elaboración propia.

Seguidamente, se preguntó a los empresarios sobre la existencia de una dependencia dedicada a actividades asociadas a I+D, diseño, control de calidad o ingeniería. El resultado reafirma la hipótesis planteada en párrafos anteriores respecto a la carencia de iniciativa o, tal vez, de capacidad innovadora del empresariado local, ya que ninguna empresa cuenta con una dependencia específica para desarrollar actividades de I+D, aunque el 20 \% afirma contar, o con un departamento de ingeniería, o con un departamento de calidad, con personal de dedicación específica. Este hallazgo es indicativo de algún esfuerzo innovador que debería dar resultados positivos a futuro.

La tabla 3 resume la información acerca de las inversiones específicas realizadas por las empresas. Es de resaltar la alta participación de la inversión en capital físico asociado a nuevos productos y procesos (no necesariamente nuevos para el mercado), capacitación tecnológica y consultorías. En nivel intermedio se encuentran los programas de aseguramiento de la calidad, gestión y administración, y un poco más abajo los de gestión ambiental. El panorama es diferente en lo que se refiere a la innovación dura, con una participación de apenas el 20 \%.

\footnotetext{
8 Premeditadamente se omitieron preguntas que hicieran referencia a montos monetarios por actividad, esto con el fin de facilitar la participación sin presiones.
} 
Tabla 3. Actividades de innovación en los últimos cinco años ( \%)

\begin{tabular}{|l|c|c|}
\hline \multicolumn{1}{|c|}{ Inversión en } & Sí & No \\
\hline $\begin{array}{l}\text { Bienes de capital que impliquen cambio tecnológico, y que estén asociados a productos } \\
\text { y procesos nuevos. }\end{array}$ & 90 & 10 \\
\hline $\begin{array}{l}\text { Licencias o acuerdos de transferencia de tecnología (patentes, marcas, secretos indus- } \\
\text { triales). }\end{array}$ & 20 & 80 \\
\hline Consultorías. & 70 & 30 \\
\hline Programas de capacitación tecnológica. & 80 & 20 \\
\hline Programas de capacitación en gestión y administración. & 60 & 40 \\
\hline Programas en control y aseguramiento de la calidad. & 60 & 40 \\
\hline Programas de gestión ambiental. & 40 & 60 \\
\hline Formas de distribución y mercadeo. & 50 & 50 \\
\hline
\end{tabular}

Fuente: elaboración propia.

Con referencia a las consultorías, el gráfico 2 muestra que estas se reparten en todas las áreas de la empresa, incluyendo las de producción, administración, finanzas, diseño y logística. Por su parte, los programas de capacitación tecnológica han tenido dos propósitos básicos para la empresa (no excluyentes entre sí): la mejora en los procesos productivos (64 \%) y el desarrollo, mejora y diseño de productos (36 \%).

Gráfico 2. Áreas en las que se hicieron las consultorías en los últimos cinco años (participación porcentual en el total de actividades)

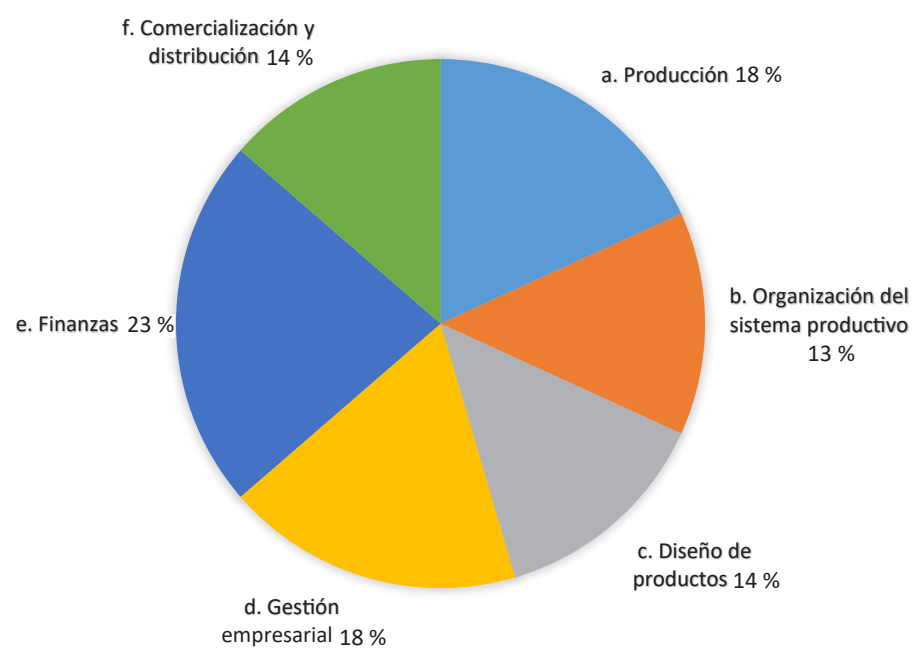

Fuente: elaboración propia. 
El gráfico 3 muestra las áreas en la que las empresas han realizado capacitaciones en gestión y administración. Se destacan las referentes a la seguridad industrial (32% de las empresas encuestadas), control de calidad y desarrollo, mejora y diseño de productos (21\% respectivamente). En la misma línea van los programas de control y aseguramiento de la calidad que han ganado popularidad como mecanismo de estandarización de la gestión y la administración eficientes. En el área de la gestión medioambiental, un grupo de empresas minoritario ha realizado esfuerzos con énfasis en la mejor utilización de insumos en los procesos de producción.

Gráfico 3. ¿En qué áreas se han llevado a cabo las capacitaciones en gestión y administración? (participación porcentual en el total de actividades)

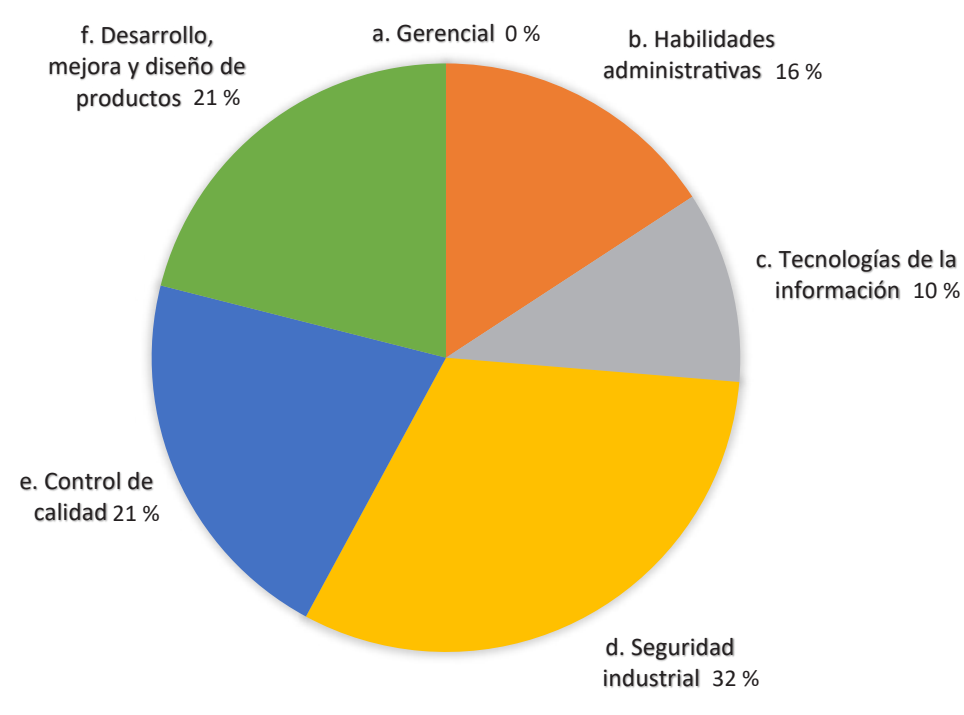

Fuente: elaboración propia.

Por último, en lo que se refiere a la distribución y el mercadeo, las encuestas revelan que la mitad de las empresas participantes ha experimentado con cambios en estas áreas en los últimos cinco años. En resumen, las actividades de innovación típicas de las empresas de la ciudad son del tipo blandas, lo que usualmente caracteriza a las empresas seguidoras con comportamiento defensivo (Vargas y Malaver, 2004; 2013), aunque del lado positivo, se puede identificar un potencial innovador esperanzador, que debe ser explotado por los gobiernos locales y regionales mediante la participación activa en un proceso de transformación incluyente, que reconozca que las universidades deben constituirse en la principal fuente de generación y difusión del conocimiento, la investigación y la innovación para las 
empresas (Vesga, 2008). Un proceso virtuoso que conlleve a la generación de empleo, ingresos y bienestar general para la sociedad.

Continuando con los resultados, se consultó a los empresarios sobre las consecuencias que han tenido las actividades de innovación en productos, procesos y organización: en productos, la frecuencia de respuesta se repartió entre creación y adaptación; en procesos, la adaptación marca la pauta con el 69 \% de frecuencia relativa; igual sucede con las actividades relacionadas con la organización, en las cuales la adaptación representa el 64 \% (gráfico 4). Nuevamente, es importante resaltar que la creación de nuevos productos, procesos y formas de organización generalmente representa una innovación para la empresa y no para el mercado. Prueba de ello es que el $67 \%$ de las empresas introdujeron nuevos productos para el mercado local, solamente el 33 \% tuvo en mente el mercado nacional, y ninguna el mercado internacional.

Gráfica 4. Resultados de las actividades de innovación en : a) productos, b) procesos y c) organización (participación porcentual)

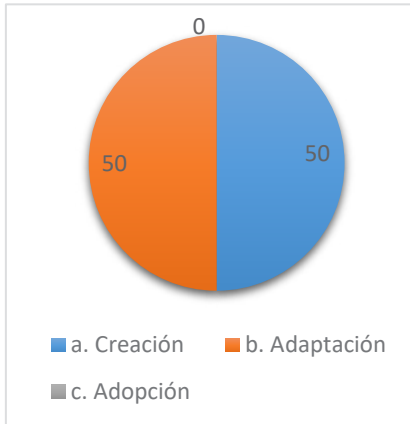

a)

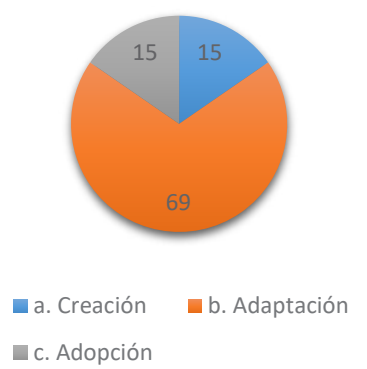

b)

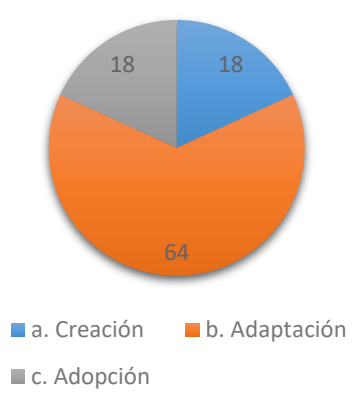

C)

Fuente: elaboración propia.

También se intentó determinar el impacto que han tenido las ACTI sobre ocho variables relevantes para la empresa (gráfico 5). Se encontró que ha sido positivo en la mayoría de los casos, sin embargo, algunos empresarios manifestaron que ha sido negativo en más de un aspecto (por frecuencia: participación de mercado y flujo de caja). Lastimosamente, no es posible estipular cuál es la causa específica de este resultado, por lo que puede ser un buen punto de partida para una futura investigación. Además, es destacable el hecho de que pocas empresas hayan generado efectos medioambientales positivos como resultado de las actividades de innovación, y sí se hayan presentado impactos negativos. 
Gráfico 5. ¿Cuál fue el impacto positivo $(\mathrm{P})$, neutro $(0)$ o negativo $(\mathrm{N})$ en los siguientes aspectos, por la introducción de innovaciones de producto, proceso u organizacionales? (participación porcentual por categoría)

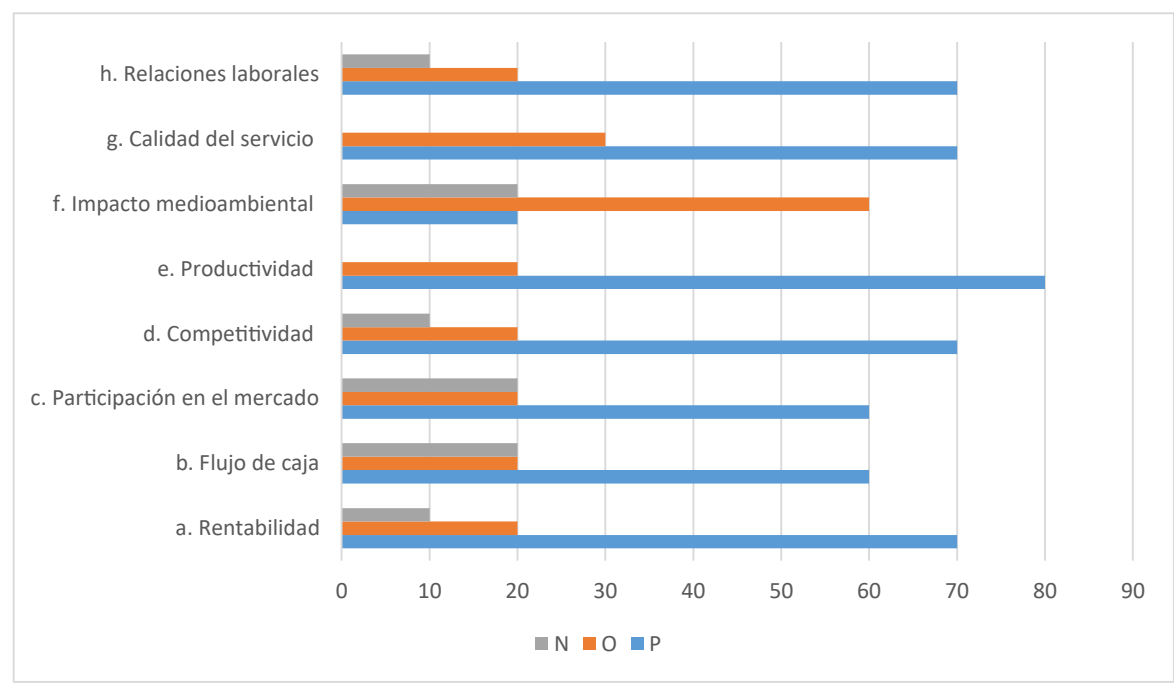

Fuente: elaboración propia.

De la misma manera, es importante conocer los porcentajes de reinversión de las empresas en actividades de innovación. El gráfico 6 recoge las evidencias al respecto. Se observa que los rangos porcentuales más representativos son 1 \% $5 \%$ y $11 \%-15 \%$. Este resultado tiene, al menos, dos connotaciones relevantes: primera, las empresas están invirtiendo parte de sus utilidades en actividades de innovación, aunque en porcentajes muy variados; segundo, las empresas pueden estar considerando positivamente las posibilidades de deducciones y descuentos que se pueden lograr por concepto de pago efectivo de impuesto de renta, con lo cual se estarían incorporando paulatinamente al SNCTi9. Según Arbeláez, Perry y Becerra (2010), la reinversión de utilidades en general, para las pymes colombianas, ha crecido sostenidamente y se situaba en un rango de $25 \%$ a 38 \% en 2008. La evidencia a nivel latinoamericano indica que, en México, la reinversión de utilidades para proyectos de investigación se situaba en el 11,3 \% y para proyectos de innovación en el 10,8 \% (Calderón, 2010); mientras que, para Argentina, la reinversión general de utilidades para empresas manufactureras promedió el $21 \%$ entre 2003 y 2012 (Cassini, 2015).

9 En Colombia la Ley 1819 de 2016 define un descuento tributario del 25 \% sobre la inversión realizada en proyectos de $\mathrm{I}+\mathrm{D}+\mathrm{i}$, siempre que estos estén articulados con universidades e institutos para el avance de la ciencia y la tecnología reconocidos por Colciencias. 
Gráfico 6. ¿Qué porcentaje de sus utilidades ha reinvertido la empresa en actividades de innovación?

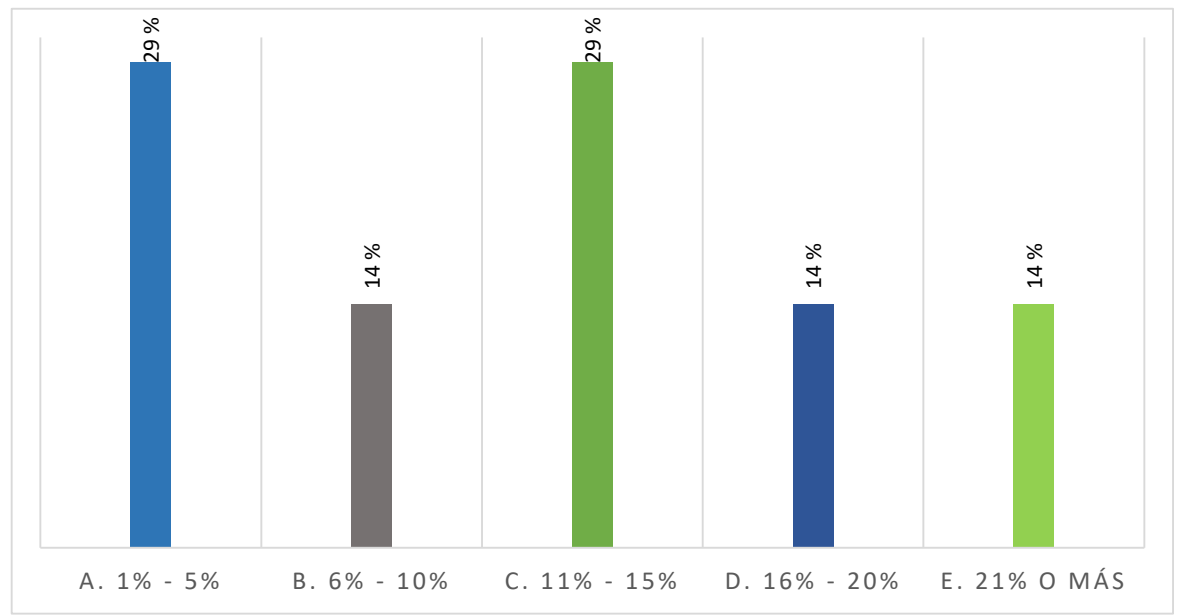

Fuente: elaboración propia.

En lo que se refiere a los objetivos de la innovación, el gráfico 7 muestra que, para el total de respuestas ordenadas, el mejorar la calidad de los productos es la razón más importante, seguido de cerca por la ampliación de mercados y la minimización de costos, mientras que, mejorar la gestión ambiental, está en último lugar de las preferencias de innovación (alguna empresa planteaba la opción de satisfacción del cliente como uno de sus objetivos). En sentido estricto, mejorar la calidad fue la primera opción para el $40 \%$ de las empresas, le siguen ampliar y conservar mercado con el $20 \%$ de las preferencias cada una. A manera de información complementaria, el 100 \% de los empresarios contestó que tienen en cuenta la innovación para establecer sus estrategias de negocio. El utilizar los objetivos de la innovación estratégicamente puede estar determinando un cambio de mentalidad de los empresarios de la ciudad frente a los retos de la supervivencia en un mundo globalizado.

Al aplicar el instrumento, también se tuvo en cuenta el problema de la financiación de las actividades de innovación, en este aspecto se pudo identificar que los recursos propios se constituyen en la principal fuente para las empresas monterianas, seguida de lejos por la banca comercial y el gobierno ${ }^{10}$ (gráfico 8). Algunos comentarios al respecto: primero, a pesar del proceso de profundización financiera que se ha dado en las ciudades colombianas, incluyendo Montería, este no parece manifestarse en la provisión amplia y oportuna de recursos para el desarrollo de proyectos de

10 Al respecto, la EDIT VIII señala que para 2016, el 79,7 \% de las empresas utilizó sus propios recursos para financiar las ACTI, el 15,8 \% utilizó recursos de la banca privada, mientras que la financiación pública correspondió solo al 0,43\%. 
tecnología e innovación, los cuales frecuentemente se asocian con el emprenderismo de base tecnológica"1. Segundo, es preocupante que los empresarios locales no aprovechen los recursos que ofrece el SNCTi en Colombia, para el desarrollo de las estrategias de innovación que dicen incorporar las empresas, tal como se mencionó anteriormente. Tercero, tampoco existe cooperación con empresas relacionadas para alcanzar objetivos comunes, minimizando costos, y no se aprovechan los recursos que se ofrecen por cooperación internacional. La cooperación es vista como algo invasivo de la privacidad de las empresas, pensamiento acorde con la idiosincrasia regional todavía prevaleciente. Al respecto, el Manual Bogotá (2001, p. 51, citando a Dini, 1996) es tajante al reconocer que "el aislamiento conspira en contra del desarrollo competitivo de las firmas".

\section{Gráfico 7. ¿Cuáles son las razones por las qué innova la empresa? (participación porcentual por categoría)}

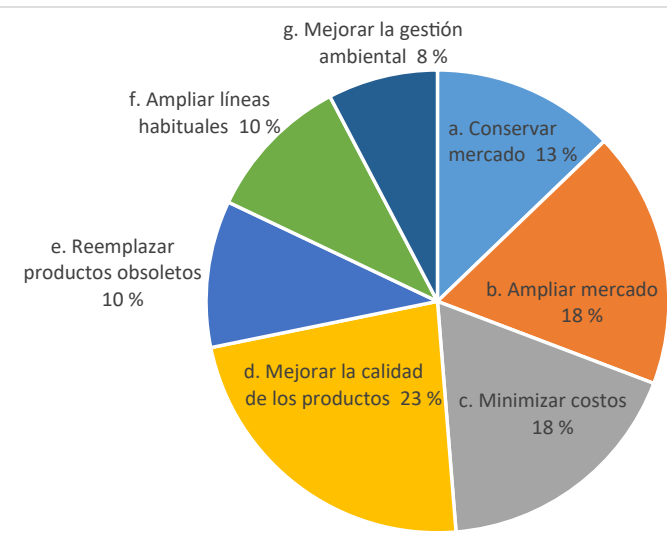

Fuente: elaboración propia.

Posteriormente, se indagó sobre las fuentes de información para las ACTi. Según el gráfico 9, los clientes se constituyen en la principal fuente para el desarrollo de ideas innovadoras (80\%), seguidos por el departamento de ventas y mercadeo, y las ideas que tengan los directivos de las empresas, que normalmente son los mismos dueños de estas (60\% respectivamente). Los proveedores y las ferias también se constituyen en una buena fuente de ideas para la innovación para la mitad de las empresas participantes. Curiosamente, a pesar de que ninguna empresa indicó que contaba con un departamento de I+D, dos empresas escogieron esta opción, haciendo referencia a los laboratorios de diseño e ingeniería. También es destacable el nulo contacto que tienen las empresas con las universidades y los centros de

11 Este planteamiento está acorde con lo señalado por González y Ramírez (s. f.) para el periodo 1981-2002. 
investigación. En lo que se refiere al orden de selección, las opciones que ocuparon los tres primeros lugares fueron: clientes, directivas y departamento de ventas y mercadeo. Los resultados están acordes con el comportamiento de empresarios seguidores que se adaptan a las necesidades específicas de los clientes sin hacer grandes esfuerzos para generar innovaciones desde adentro, característica definitoria de los empresarios innovadores en sentido estricto.

Gráfico 8. ¿Cuáles son las principales fuentes de financiamiento utilizadas por la empresa para realizar las actividades de innovación? (participación porcentual por categoría)

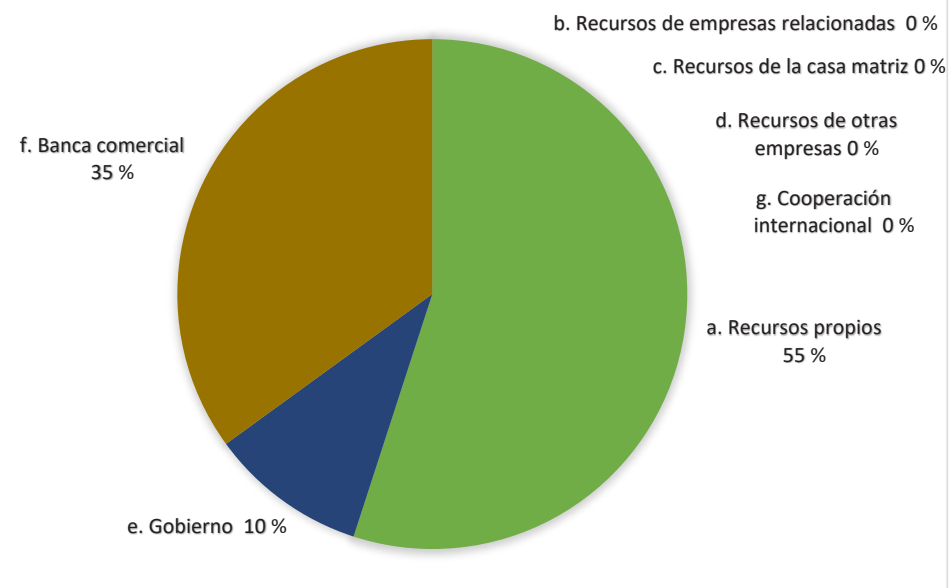

Fuente: elaboración propia.

Gráfico 9. ¿Cuáles son las principales fuentes (internas y externas) de información utilizadas por la empresa? (participación porcentual por categoría)

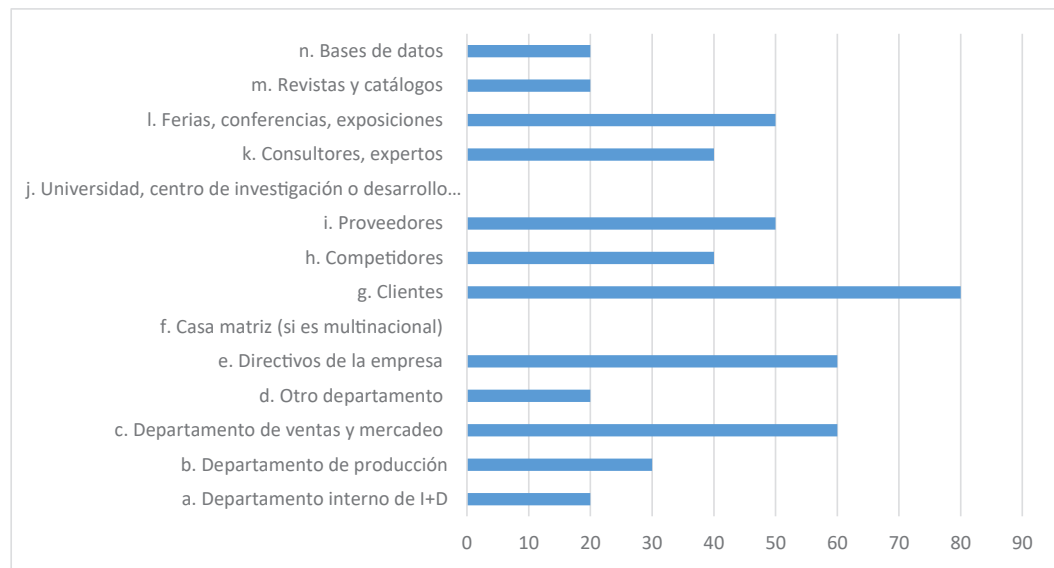

Fuente: elaboración propia. 
En último lugar, se consultó a los empresarios sobre los factores que afectan la innovación. Según el gráfico 10, la principal preocupación la constituye el riesgo económico con el $60 \%$, seguido por la carencia de recursos propios y las fuentes de financiación con el 40 \% respectivamente. En cuanto al orden de selección, la carencia de recursos propios, las fuentes de financiación y el riesgo económico constituyen las opciones más significativas y ocupan los tres primeros lugares de la lista. El riesgo económico tiene que ver con la aversión que caracteriza a las empresas no innovadoras o seguidoras, probablemente tenga que ver con las fuentes de financiación y su disponibilidad. La carencia de recursos propios se puede relacionar con la rentabilidad del negocio y sus posibilidades de generación de beneficios económicos, pero también con la reinversión de utilidades que se comentó anteriormente. En definitiva, son factores que están interrelacionados y que no se pueden entender como eslabones sueltos de una cadena potencial, por lo tanto, deben ser tenidos en cuenta para la aplicación de las políticas correctas a nivel de sistema nacional y de sistema regional, recordando que la empresa es el lugar en donde se plasma la innovación. En palabras de Mendizábal (2002, p. 108), los gobiernos deben "crear un entorno favorable que facilite la innovación empresarial".

Gráfico 10. ¿Cuáles son los problemas que enfrenta la empresa para realizar sus proyectos de innovación? (participación porcentual por categoría)

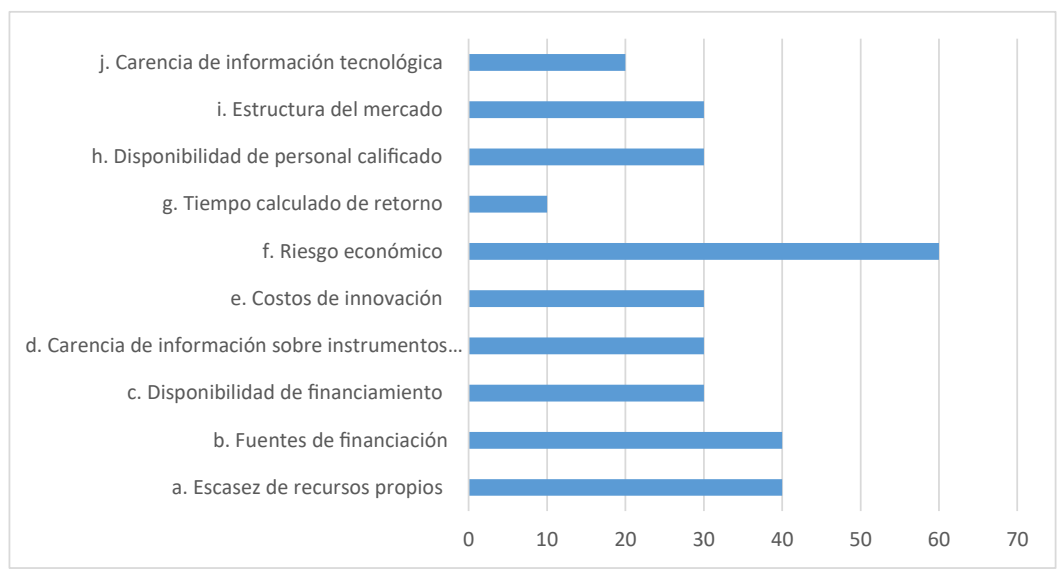

Fuente: elaboración propia.

\section{CONCLUSIONES}

Este trabajo ha reafirmado lo que otros estudios latinoamericanos, nacionales y regionales han señalado con anterioridad: la industria manufacturera es marginalmente innovadora en nuestro entorno, y lo que sucede con el empresariado de la ciudad de 
Montería es un reflejo de lo que ocurre a nivel nacional. Se identifica un alto grado de informalidad en los procesos de innovación y poco desarrollo de capacidades tecnológicas, tal como señalan Vargas y Malaver (2013). En general las ACTi que realizan las empresas incluyen la incorporación de capital físico, capacitaciones en diferentes áreas y las certificaciones de calidad. Las actividades relacionadas con I + D para el desarrollo de nuevos o mejores productos es incipiente y se relaciona con innovación para la empresa y no para el mercado.

Las empresas locales presentan comportamientos de carácter defensivo que no se corresponden con el escenario de internacionalización asumido por el país desde principios de los noventa. Con todo, se verifican algunos comportamientos prometedores que permiten ser optimistas en el mediano y largo plazo, siempre que se logre una integración horizontal de objetivos, desde los gobiernos locales y regionales, en conjunto con las universidades y los centros de desarrollo tecnológico.

Hay que reconocer que, para alcanzar el objetivo de conseguir y consolidar un sistema regional de innovación en el mediano plazo, debe generarse confianza entre los diversos actores participantes: los gobiernos deben liderar el proceso con transparencia, por encima de coyunturas políticas y de colores partidistas. Los centros de pensamiento deben volcar sus capacidades humanas para ayudar en la solución de los diversos problemas que se presentan, siempre con un alto compromiso social. Y los empresarios, como actores principales, deben ser conscientes de que las ganancias de productividad y competitividad necesarias para crecer y sobrevivir en mercados evolutivos, solamente serán posibles y sostenibles con cooperación, integración y apertura. Se hace necesario apoyar el aprendizaje secuencial para transformar una visión empresarial heterogénea.

Queda en el aire el interrogante formulado por Gómez y Mitchell (2014), sobre ¿Cómo lograr que las empresas se conviertan en el centro del sistema de ciencia, tecnología e innovación?

Finalmente, se hace necesario que este tipo de ejercicios de investigación tenga continuidad en el tiempo y se amplíe el campo de observación, por lo que se propone la participación activa de los gremios, de las universidades y de los gobiernos, con el fin de consolidar un sistema de información sobre la innovación desde lo regional y lo local.

\section{BIBLIOGRAFÍA}

Aghion, Philippe y Howitt, Peter (1999). Endogenous growth theory. Cambridge: MIT Press, 694 p. Albornoz, Mario (2009). Indicadores de innovación: las dificultades de un concepto en evolución. En: Revista Iberoamericana de Ciencia, Tecnología y Sociedad -CTS, vol. 5, n. ${ }^{\circ}$ 13, p. 9-25. 
Anlló, Guillermo y Suárez, Diana (2008). Innovación: algo más que I+D. Evidencias Iberoamericanas a partir de las encuestas de innovación: construyendo las estrategias empresarias competitivas. En: Red Iberoamericana de Indicadores de Ciencia y Tecnología -Ricyt (Ed.), El estado de la ciencia 2008: principales indicadores de ciencia y tecnología iberoamericanos. Buenos Aires: Ricyt, p. 73-103.

Anlló, Guillermo; Suárez, Diana y De Angelis, Jesica (2009). Indicadores de innovación en América Latina: 10 años del Manual de Bogotá. En: Red Iberoamericana de Indicadores de Ciencia y Tecnología -Ricyt (Ed.), El estado de la ciencia 2009: principales indicadores de ciencia y tecnología iberoamericanos. Buenos Aires: Ricyt, p. 99-124.

Arbeláez, María y Parra, Mónica (2011). Innovation, RED Investment and Productivity in Colombian firms. Washington: Inter-American Development Bank, $53 \mathrm{p}$.

Arbeláez, María; Perry, Guillermo y Becerra, Alejandro (2010). Estructura de financiamiento y restricciones financieras de las empresas en Colombia. Caracas: Banco de Desarrollo de América Latina -CAF, 56 p.

Barro, Robert (1991). Economic growth in a cross section of countries. En: The Quarterly Journal of Economics, vol. 106, n. ${ }^{\circ}$ 2, p. 407-443.

Barro, Robert y Sala-i-Martin, Xavier (1999). Economic growth. Cambridge: MIT Press, 539 p.

Bitrán, Eduardo; Benavente, José y Maggi, Claudio (2011). Bases para una estrategia de innovación y competitividad para Colombia. Santiago de Chile: Centro de Productividad Universidad Adolfo Ibáñez, 36 p.

Bonilla, Manuel (2000). Cambio tecnológico y crecimiento en la industria manufacturera colombiana (1990 1996). En: Barómetro, vol. 1, n. 2, 8 p.

Calderón, Eduardo (2010). Retos de la vinculación en México y avances en la estrategia de la SES. Recuperado de http://www.tecnm.mx/vinculacion/memoria-de-la-reunion-nacional-de-vinculacion-2010

Cassini, Lorenzo (2015). Reinversión de utilidades y concentración en la industria manufacturera argentina en la posconvertibilidad. En: Revista Realidad Económica, n.o 292, p. 44-63.

Consejo Privado de Competitividad (2018). Ciencia, tecnología e innovación. En: Barragán, Andrés (Ed.), Informe nacional de competitividad 2017-2018. Bogotá: Zetta Comunicadores, p. 220-247.

DANE -Departamento Administrativo Nacional de Estadística (2017). Metodología general encuesta de desarrollo e innovación tecnológica en la industria manufacturera -EDIT. Bogotá: DANE, 50 p.

DANE -Departamento Administrativo Nacional de Estadística, DNP -Departamento Nacional de Planeación y Colciencias (2006). Innovación y desarrollo tecnológico en la industria manufacturera: Colombia 2003-2004. Bogotá: DANE, 198 p.

Dini, Marco y Stumpo, Giovanni (2011). Políticas para la innovación de las pequeñas y medianas empresas en América Latina. En: Dini, Marco y Stumpo, Giovanni (Comps.), Colección documentos de proyectos. Santiago de Chile: Cepal, p.11-34. 
DNP -Departamento Nacional de Planeación (2007). Política nacional para la transformación productiva y la promoción de las micro, pequeñas y medianas empresas: un esfuerzo público-privado. Bogotá: Consejo Nacional de Política Económica y Social -Conpes, 31 p.

DNP -Departamento Nacional de Planeación (2008). Política nacional de competitividad y productividad. Bogotá: Consejo Nacional de Política Económica y Social -Conpes, 83 p.

DNP -Departamento Nacional de Planeación (2009). Política nacional de ciencia, tecnología e innovación. Bogotá: Consejo Nacional de Política Económica y Social -Conpes, 68 p.

DNP -Departamento Nacional de Planeación (2010). Informe de seguimiento a la política nacional de competitividad y productividad: Ley 1253 de 2008. Bogotá: Consejo Nacional de Política Económica y Social -Conpes, 143 p.

DNP - Departamento Nacional de Planeación (2015a). Lineamiento de política para estimular la inversión privada en ciencia, tecnología e innovación a través de deducciones tributarias. Bogotá: Consejo Nacional de Política Económica y Social -Conpes, 54 p.

DNP -Departamento Nacional de Planeación (2015b). Política nacional de ciencia, tecnología e innovación 2015-2025. Bogotá: Consejo Nacional de Política Económica y Social -Conpes, $161 \mathrm{p}$.

DNP -Departamento Nacional de Planeación (2017). Actualización del Documento Conpes 3834: Lineamientos de política para estimular la inversión privada en ciencia, tecnología e innovación a través de deducciones tributarias. Bogotá: Consejo Nacional de Política Económica y Social -Conpes, 30 p.

Durán, Xavier; Ibáñez, Rodrigo; Salazar, Mónica y Vargas, Marisela (1998). La innovación tecnológica en Colombia: características por tamaño y tipo de empresa. En: Planeación y Desarrollo, vol. 29, n. ${ }^{\circ}$ 1, p. 15-71.

Durán, Xavier; Ibáñez, Rodrigo; Salazar, Mónica y Vargas, Marisela (2000). La innovación tecnológica en Colombia: características por sector industrial y región geográfica. Bogotá: Observatorio Colombiano de Ciencia y Tecnología -OCyT, 198 p.

Esguerra, Carolina; Castro, Juan y González, Néstor (s.f.). Cambio estructural y competitividad: el caso colombiano. Bogotá: DANE, 45 p.

Fagerberg, Jan (2005). Innovation a guide to the literature. En: Fagerberg, Jan, Mowery, David y Nelson, Richard (Eds.), The Oxford Handbook of innovation. Oxford: Oxford University Press, p. 1-26.

Freeman, Chris (1995). The National System of Innovation in historical perspective. En: Cambridge Journal of Economics, vol. 19, n. ${ }^{\circ}$ 1, p. 5-24.

Godin, Benoît (2002). The rise of innovation surveys: measuring a fuzzy concept. Canadá: History and Sociology of STI Statistics.

Gómez, Fredy (2009). Las capacidades de innovación tecnológica y el desempeño empresarial y sectorial en Colombia. Trabajo de grado para optar al título de Magíster en Ingeniería Administrativa, Maestría en Ingeniería Administrativa, Universidad Nacional de Colombia, Medellín, $51 \mathrm{p}$. 
Gómez, María (2011). Evolución de las capacidades de innovación en la industria colombiana: Un análisis comparativo de los resultados de las encuestas de innovación 1996 y 2005. Trabajo de grado para optar al título de Magíster en Ingeniería Administrativa, Maestría en Ingeniería Administrativa, Universidad Nacional de Colombia, Medellín, 58 p.

Gómez, Hernando y Mitchell, Daniel (2014). Innovación y emprendimiento en Colombia: balance, perspectivas y recomendaciones de política, 2014-2018. Bogotá: Cuadernos de Fedesarrollo, $82 \mathrm{p}$.

González, Néstor y Ramírez, Patricia (s. f.). Cambio estructural y competitividad en los departamentos colombianos. Bogotá: DANE, 40 p.

Guillard, Charlotte y Salazar, Mónica (2017). La experiencia en encuestas de innovación de algunos países latinoamericanos. Washington: Banco Interamericano de Desarrollo, 15 p.

Howitt, Peter y Ha, Joon Kyung (2007). Accounting for trends in productivity and RED: a Schumpeterian critique of Semi Endogenous Growth Theory. En: Journal of Money, Credit and Banking, vol. 39, n. ${ }^{\circ}$, p. 733-774.

Jaramillo, Hernán; Lugones, Gustavo y Salazar, Mónica (2001). Normalización de indicadores de innovación tecnológica en América Latina y el Caribe. Manual de Bogotá. Recuperado de http://www.ricyt.org/manuales/doc_view/5-manual-de-bogota

Jones, Charles y Romer, Paul (2009). The new Kaldor facts, ideas, institutions, population, and human capital. En: American Economic Journal: Macroeconomics, vol. 2, n. ${ }^{\circ}$ 1, p. 224-245.

Katz, Jorge (1990). Teoría económica y política tecnológica: conceptos generales de gestión tecnológica. Santiago de Chile: Banco Interamericano de Desarrollo, p. 49-69.

Katz, Jorge (2000). Reformas estructurales, productividad y conducta tecnológica en América Latina. Chile: Cepal, 221 p.

Langebaek, Andrés y Vásquez, Diego (2007). Determinantes de la actividad innovadora en la industria manufacturera colombiana. En: Borradores de Economía, n. 433, 36 p.

Lundvall, Bengt-Åke (2016). The learning economy and the economics of hope. Londres: Anthem Press, $406 \mathrm{p}$.

Marins, Luciana; Anlló, Guillermo y Schaaper, Martín (2013). Estadísticas de innovación: el desafío de la comparabilidad. En: Red Iberoamericana de Indicadores de Ciencia y Tecnología -RICYT (Ed.), El estado de la ciencia 2012: principales indicadores de ciencia y tecnología iberoamericanos. Buenos Aires: RICYT, p. 65-79.

Mendizábal, Guillermo (2002). Las estrategias para la innovación tecnológica en Castilla y León. Trabajo de grado para optar al título de Doctor en Ciencias Económicas y Empresariales, Universidad de Valladolid, Valladolid, 453 p.

Nelson, Richard (1995). Recent evolutionary theorizing about economic change. En: Journal of Economic Literature, vol. 33, n. ${ }^{\circ}$ 1, p. 48-90.

Nelson, Richard (2008). Economic Development from the Perspective of Evolutionary Economic Theory. En: Oxford Development Studies, vol. 36, n. ${ }^{\circ}$ 1, p. 9-21. 
Nelson, Richard y Winter, Sidney (1982). An evolutionary theory of economic change. Cambridge: Belknap Press of Harvard, 437 p.

Nelson, Richard y Winter, Sidney (2002). Evolutionary theorizing in economics. En: Journal of Economic Perspectives, vol. 16, n. ${ }^{\circ}$ 2, p. 23-46.

OCDE - Organización para la Cooperación y el Desarrollo Económicos (2005). Oslo Manual: proposed guidelines for collecting and interpreting technological innovation data, the measurement of scientific and technological activities. París: OCDE, 163 p.

OCDE - Organización para la Cooperación y el Desarrollo Económicos (2013). Estudios de la OCDE de las políticas de innovación: Colombia, evaluación general y recomendaciones. Bogotá: OCDE, 43 p.

OCDE - Organización para la Cooperación y el Desarrollo Económicos (2015). Frascati Manual 2015: guidelines for collecting and reporting data on research and experimental development, the measurement of scientific, technological and innovation activities. París: OCDE, 398 p.

Pavitt, Keith (2005). Innovation processes. En: Fagerberg, Jan, Mowery, David y Nelson, Richard (Eds.), The Oxford Handbook of innovation. Oxford: Oxford University Press, p. 86-114.

Pavitt, Keith y Bell, Martin (1992). Accumulating technological capability in developing countries. En: The World Bank Economic Review, vol. 6, p. 257-281.

Ramírez, Diego; Arboleda, Jaime y Robledo, Jorge (1999). Indicadores para el Sistema Regional de Innovación de Antioquia. En: Revista de Ciência e Tecnologia Política e Gestão para a Periferia -RECITEC, vol. 3, n. ${ }^{\circ}$ 2, p. 291-307.

Romer, Paul (1990). Endogenous technological change. En: Journal of Political Economy, vol. 98, n. ${ }^{0}$ 5, p. $71-102$.

Romer, Paul (1994). The origins of endogenous growth. En: Journal of Economic Perspectives, vol. 8, n. $^{\circ}$ 1, p. 3-22.

Schumpeter, Joseph (1946). Capitalismo, socialismo y democracia. Barcelona: Folio, 512 p.

Torres, Jairo; Cataño, Gabriel y Arias, José (2015). Caracterización de la innovación en las pymes manufactureras en Colombia desde la perspectiva del análisis clúster. En: U.D.C.A Actualidad E Divulgación Científica, vol. 18, n. ${ }^{\circ}$ 2, p. 525-532.

Vargas, Marisela y Malaver, Florentino (2004). Los avances en la medición del desarrollo tecnológico en la industria colombiana. En: Revista CTS, vol. 1, n. ${ }^{\circ}$ 2, p. 137-166.

Vargas, Marisela y Malaver, Florentino (2013). Formas de innovar y sus implicaciones de política: lecciones de una experiencia. En: Cuadernos de Economía, vol. 32, n. ${ }^{\circ}$ 60, p. 537-570.

Vesga, Rafael (2008). Emprendimiento e Innovación en Colombia ¿Qué nos está haciendo falta? Bogotá: Universidad de los Andes, 16 p. 